\title{
Transcendental submanifolds of projective space
}

\author{
Wojciech Kucharz*
}

\begin{abstract}
Given integers $m$ and $c$ satisfying $m-2 \geq c \geq 2$, we explicitly construct a nonsingular $m$-dimensional algebraic subset of $\mathbb{P}^{m+c}(\mathbb{R})$ that is not isotopic to the set of real points of any nonsingular complex algebraic subset of $\mathbb{P}^{m+c}(\mathbb{C})$ defined over $\mathbb{R}$. The first examples of this type were obtained by Akbulut and King in a more complicated and nonconstructive way, and only for certain large integers $m$ and $c$.
\end{abstract}

Mathematics Subject Classification (2000). 57R55,14P25.

Keywords. Smooth manifold, algebraic set, isotopy.

\section{Introduction}

Denote by $\mathbb{P}^{n}(\mathbb{R})$ and $\mathbb{P}^{n}(\mathbb{C})$ real and complex projective $n$-spaces. We regard $\mathbb{P}^{n}(\mathbb{R})$ as a subset of $\mathbb{P}^{n}(\mathbb{C})$. A smooth (of class $\ell^{\infty}$ ) submanifold $M$ of $\mathbb{P}^{n}(\mathbb{R})$ is said to be of algebraic type if it is isotopic in $\mathbb{P}^{n}(\mathbb{R})$ to the set of real points of a nonsingular complex algebraic subset of $\mathbb{P}^{n}(\mathbb{C})$ defined over $\mathbb{R}$; otherwise $M$ is said to be transcendental. It is not at all obvious that transcendental submanifolds exist. However, Akbulut and King [2] proved the existence of transcendental submanifolds $M$ of $\mathbb{P}^{n}(\mathbb{R})$ which can even be realized as nonsingular algebraic subsets of $\mathbb{P}^{n}(\mathbb{R})$. Their examples are obtained in a nonconstructive way, by a method which requires both $m=\operatorname{dim} M$ and $n-m$ to be large integers satisfying $2 m-n \geq 2$. In the present paper we explicitly construct such examples, assuming only $n-m \geq 2$ and $2 m-n \geq 2$. Moreover, we verify that $M$ is a transcendental submanifold of $\mathbb{P}^{n}(\mathbb{R})$ using only the Barth-Larsen theorem [6, Corollary 6.5] and completely avoiding all results of [1], [2]. More precisely, denote by $S^{k}$ the unit $k$-sphere,

$$
S^{k}=\left\{\left(y_{1}, \ldots, y_{k+1}\right) \in \mathbb{R}^{k+1} \mid y_{1}^{2}+\cdots+y_{k+1}^{2}=1\right\} .
$$

In Section 3 we prove the following:

\footnotetext{
* The paper was completed at the Max-Planck-Institut für Mathematik in Bonn, whose support and hospitality are gratefully acknowledged.
} 
Theorem 1.1. Let $m$ and $n$ be positive integers satisfying $n-m \geq 2$ and $2 m-n \geq 2$. Let

$$
\varphi: \mathbb{P}^{2}(\mathbb{R}) \times S^{m-2} \longrightarrow \mathbb{P}^{n}(\mathbb{R})
$$

be defined by

$$
\begin{aligned}
& \varphi\left(\left(x_{1}: x_{2}: x_{3}\right),\left(y_{1}, \ldots, y_{m-1}\right)\right) \\
& \quad=\left(x_{1}^{2}+x_{2}^{2}+x_{3}^{2}: x_{1} x_{2}: x_{1} x_{3}: x_{2} x_{3}: \sigma y_{1}: \ldots: \sigma y_{m-1}: 0: \ldots: 0\right),
\end{aligned}
$$

where 0 is repeated $n-m-2$ times and $\sigma=x_{1}^{2}+2 x_{2}^{2}+3 x_{3}^{2}$. Then:

(i) The image $M=\varphi\left(\mathbb{P}^{2}(\mathbb{R}) \times S^{m-2}\right)$ is an m-dimensional nonsingular algebraic subset of $\mathbb{P}^{n}(\mathbb{R})$.

(ii) $\varphi: \mathbb{P}^{2}(\mathbb{R}) \times S^{m-2} \rightarrow M$ is a biregular isomorphism.

(iii) $M$ is a transcendental submanifold of $\mathbb{P}^{n}(\mathbb{R})$.

It follows directly from Theorem 1.1 that for any integers $m$ and $c$ satisfying $m-2 \geq c \geq 2$, there is a nonsingular algebraic set $M$ in $\mathbb{P}^{m+c}(\mathbb{R})$ such that $\operatorname{dim} M=m$ and $M$ is a transcendental submanifold. In particular, there are transcendental submanifolds of arbitrary dimension $m \geq 4$. The existence of transcendental submanifolds of dimension 2 or 3 remains unsettled at this time. There are no transcendental submanifolds of dimension 1 or of codimension 1 . The last assertion is a special case of the following well known fact.

Remark 1.2. Let $M$ be a smooth $m$-dimensional submanifold of $\mathbb{P}^{n}(\mathbb{R})$. If either $n-m=1$ or $2 m+1 \leq n$, then there exists a smooth embedding $e: M \rightarrow \mathbb{P}^{n}(\mathbb{R})$, arbitrarily close in the $\mathcal{C}^{\infty}$ topology to the inclusion map $M \hookrightarrow \mathbb{P}^{n}(\mathbb{R})$, such that $e(M)$ is the set of real points of a nonsingular complex algebraic subset of $\mathbb{P}^{n}(\mathbb{C})$ defined over $\mathbb{R}$.

If $n-m=1$, the claim is explicitly established for example in [3, Theorem 7.1]. For the second case, consider $\mathbb{P}^{n}(\mathbb{R})$ as a subset of $\mathbb{P}^{k}(\mathbb{R})$, where $k$ is a large integer. By [8], there exists a smooth embedding $j: M \rightarrow \mathbb{P}^{k}(\mathbb{R})$, arbitrarily close in the $\bigodot^{\infty}$ topology to the inclusion map $M \hookrightarrow \mathbb{P}^{k}(\mathbb{R})$, such that $j(M)$ is a nonsingular algebraic subset of $\mathbb{P}^{k}(\mathbb{R})$. Increasing $k$ if necessary and making use of Hironaka's resolution of singularities theorem [7], we may assume that the Zariski complex closure of $j(M)$ in $\mathbb{P}^{k}(\mathbb{C})$ is nonsingular. If $2 m+1 \leq n$, we obtain an embedding $e: M \rightarrow \mathbb{P}^{n}(\mathbb{R})$ with the required properties by composing $j$ with an appropriate generic projection onto $\mathbb{P}^{n}(\mathbb{R})$. 


\section{A criterion for transcendence}

First we need some results related to the Picard group. Following the current custom, we state them in the language of schemes.

Let $V$ be a smooth projective scheme over $\mathbb{R}$. Assume that the set $V(\mathbb{R})$ of $\mathbb{R}$ rational points of $V$ is nonempty. We regard $V(\mathbb{R})$ as a compact smooth manifold. Every invertible sheaf $\mathscr{L}$ on $V$ determines a real line bundle on $V(\mathbb{R})$, denoted $\mathscr{L}(\mathbb{R})$. The correspondence which assigns to each invertible sheaf $\mathscr{L}$ on $V$ the first StiefelWhitney class $w_{1}(\mathscr{L}(\mathbb{R}))$ of $\mathscr{L}(\mathbb{R})$ gives rise to a canonical homomorphism

$$
w_{1}: \operatorname{Pic}(V) \longrightarrow H^{1}(V(\mathbb{R}), \mathbb{Z} / 2),
$$

defined on the Picard group $\operatorname{Pic}(V)$ of isomorphism classes of invertible sheaves on $V$. We set

$$
H_{\text {alg }}^{1}(V(\mathbb{R}), \mathbb{Z} / 2)=w_{1}(\operatorname{Pic}(V)) .
$$

It will be convenient to recall another description of $\operatorname{Pic}(V)$. Consider the scheme $V_{\mathbb{C}}=V \times_{\mathbb{R}} \mathbb{C}$ over $\mathbb{C}$ and its Picard group $\operatorname{Pic}\left(V_{\mathbb{C}}\right)$. The Galois group $G=$ $\operatorname{Gal}(\mathbb{C} / \mathbb{R})$ of $\mathbb{C}$ over $\mathbb{R}$ acts on $\operatorname{Pic}\left(V_{\mathbb{C}}\right)$. We denote by $\operatorname{Pic}\left(V_{\mathbb{C}}\right)^{G}$ the subgroup of $\operatorname{Pic}\left(V_{\mathbb{C}}\right)$ consisting of the elements fixed by $G$. Given an invertible sheaf $\mathscr{L}$ on $V$, we write $\mathscr{L}_{\mathbb{C}}$ for the corresponding sheaf on $V_{\mathbb{C}}$. The correspondence $\mathscr{L} \rightarrow \mathscr{L}_{\mathbb{C}}$ defines a canonical group homomorphism

$$
\alpha: \operatorname{Pic}(V) \longrightarrow \operatorname{Pic}\left(V_{\mathbb{C}}\right)^{G} .
$$

It follows from the general theory of descent [4] that $\alpha$ is an isomorphism (a simple treatment of the case under consideration can also be found in [5]).

As usual, we set $\mathbb{P}_{\mathbb{R}}^{n}=\operatorname{Proj}\left(\mathbb{R}\left[T_{0}, \ldots, T_{n}\right]\right)$ and identify $\mathbb{P}_{\mathbb{R}}^{n}(\mathbb{R})$ with $\mathbb{P}^{n}(\mathbb{R})$. Thus if $V$ is a subscheme of $\mathbb{P}_{\mathbb{R}}^{n}$, then $V(\mathbb{R})$ is a subset of $\mathbb{P}^{n}(\mathbb{R})$.

Proposition 2.1. Let $V$ be a closed smooth $m$-dimensional subscheme of $\mathbb{P}_{\mathbb{R}}^{n}$. If $2 m-n \geq 2$, then

$$
H_{\mathrm{alg}}^{1}(V(\mathbb{R}), \mathbb{Z} / 2)=i^{*}\left(H^{1}\left(\mathbb{P}^{n}(\mathbb{R}), \mathbb{Z} / 2\right)\right),
$$

where $i: V(\mathbb{R}) \hookrightarrow \mathbb{P}^{n}(\mathbb{R})$ is the inclusion map.

Proof. Let $j: V \hookrightarrow \mathbb{P}_{\mathbb{R}}^{n}$ and $j_{\mathbb{C}}: V_{\mathbb{C}} \hookrightarrow \mathbb{P}_{\mathbb{C}}^{n}=\mathbb{P}_{\mathbb{R}}^{n} \times_{\mathbb{R}} \mathbb{C}$ be the inclusion morphisms. By the Barth-Larsen theorem [6, Corollary 6.5], the induced homomorphism

$$
j_{\mathbb{C}}^{*}: \operatorname{Pic}\left(\mathbb{P}_{\mathbb{C}}^{n}\right) \longrightarrow \operatorname{Pic}\left(V_{\mathbb{C}}\right)
$$

is an isomorphism. Since $j_{\mathbb{C}}^{*}$ is $G$-equivariant, the restriction

$$
j_{\mathbb{C}}^{*}: \operatorname{Pic}\left(\mathbb{P}_{\mathbb{C}}^{n}\right)^{G} \longrightarrow \operatorname{Pic}\left(V_{\mathbb{C}}\right)^{G}
$$


is an isomorphism. We have the following commutative diagram:

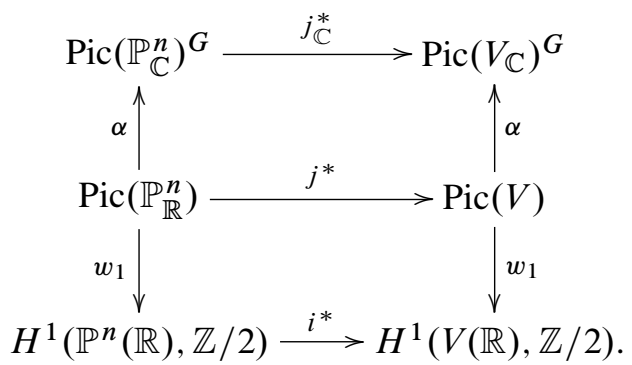

Since the homomorphisms $\alpha$ are isomorphisms and

$$
H^{1}\left(\mathbb{P}^{n}(\mathbb{R}), \mathbb{Z} / 2\right)=H_{\text {alg }}^{1}\left(\mathbb{P}^{n}(\mathbb{R}), \mathbb{Z} / 2\right),
$$

it follows that

$$
H_{\mathrm{alg}}^{1}(V(\mathbb{R}), \mathbb{Z} / 2)=i^{*}\left(H^{1}\left(\mathbb{P}^{n}(\mathbb{R}), \mathbb{Z} / 2\right)\right),
$$

as required.

Note that a smooth submanifold of $\mathbb{P}^{n}(\mathbb{R})$ is of algebraic type if and only if it is isotopic in $\mathbb{P}^{n}(\mathbb{R})$ to $V(\mathbb{R})$ for some closed smooth subscheme $V$ of $\mathbb{P}_{\mathbb{R}}^{n}$. Hence Proposition 2.1 yields the following criterion for transcendence.

Proposition 2.2. Let $M$ be a compact smooth $m$-dimensional submanifold of $\mathbb{P}^{n}(\mathbb{R})$. Assume that the inclusion map e: $M \hookrightarrow \mathbb{P}^{n}(\mathbb{R})$ induces a trivial homomorphism

$$
e^{*}: H^{1}\left(\mathbb{P}^{n}(\mathbb{R}), \mathbb{Z} / 2\right) \longrightarrow H^{1}(M, \mathbb{Z} / 2),
$$

that is, $e^{*}=0$. If $M$ is nonorientable and $2 m-n \geq 2$, then $M$ is a transcendental submanifold of $\mathbb{P}^{n}(\mathbb{R})$.

Proof. Suppose to the contrary that $M$ is of algebraic type. Let $V$ be a closed smooth subscheme of $\mathbb{P}_{\mathbb{R}}^{n}$ with $V(\mathbb{R})$ isotopic to $M$ in $\mathbb{P}^{n}(\mathbb{R})$. Then the homomorphism

$$
i^{*}: H^{1}\left(\mathbb{P}^{n}(\mathbb{R}), \mathbb{Z} / 2\right) \longrightarrow H^{1}(V(\mathbb{R}), \mathbb{Z} / 2),
$$

induced by the inclusion map $i: V(\mathbb{R}) \hookrightarrow \mathbb{P}^{n}(\mathbb{R})$, is trivial. Since $\operatorname{dim} V=m$ and $2 m-n \geq 2$, Proposition 2.1 implies

$$
H_{\text {alg }}^{1}(V(\mathbb{R}), \mathbb{Z} / 2)=0 .
$$

On the other hand, the first Stiefel-Whitney class $w_{1}(V(\mathbb{R}))$ of $V(\mathbb{R})$ is nonzero, $V(\mathbb{R})$ being a nonorientable manifold. Moreover, $w_{1}(V(\mathbb{R}))=w_{1}(\mathcal{K}(\mathbb{R}))$, where $\mathcal{K}$ is the canonical invertible sheaf of $V$, and hence, $w_{1}(V(\mathbb{R}))$ is in $H_{\mathrm{alg}}^{1}(V(\mathbb{R}), \mathbb{Z} / 2)$. In view of this contradiction, the proof is complete. 


\section{Transcendental submanifolds}

We begin with some preliminary observations. Identify $\mathbb{R}^{n}$ with its image under the map

$$
\mathbb{R}^{n} \longrightarrow \mathbb{P}^{n}(\mathbb{R}), \quad\left(x_{1}, \ldots, x_{n}\right) \longmapsto\left(1: x_{1}: \ldots: x_{n}\right) ;
$$

thus $\mathbb{R}^{n} \subset \mathbb{P}^{n}(\mathbb{R})$. An algebraic subset $X$ of $\mathbb{R}^{n}$ is said to be projectively closed if $X$ is also an algebraic subset of $\mathbb{P}^{n}(\mathbb{R})$. One readily checks that $X$ is projectively closed if and only if it can be defined by a real polynomial equation

$$
f\left(x_{1}, \ldots, x_{n}\right)=0,
$$

where the homogeneous form of top degree in $f$ vanishes only at 0 in $\mathbb{R}^{n}$.

Lemma 3.1. Let $X$ be an algebraic subset of $\mathbb{R}^{k}$ contained in the open half-space

$$
H=\left\{\left(x_{1}, \ldots, x_{k}\right) \in \mathbb{R}^{k} \mid x_{k}>0\right\} .
$$

Then the map $\psi: X \times S^{\ell} \rightarrow \mathbb{R}^{k+\ell}$ defined by

$$
\psi\left(\left(x_{1}, \ldots, x_{k}\right),\left(y_{1}, \ldots, y_{\ell+1}\right)\right)=\left(x_{1}, \ldots, x_{k-1}, x_{k} y_{1}, \ldots, x_{k} y_{\ell+1}\right)
$$

is an algebraic embedding, that is, the image $Y=\psi\left(X \times S^{\ell}\right)$ is an algebraic subset of $\mathbb{R}^{k+\ell}$ and $\psi: X \times S^{\ell} \rightarrow Y$ is a biregular isomorphism. Moreover, if $X$ is projectively closed in $\mathbb{R}^{k}$, then $Y$ is projectively closed in $\mathbb{R}^{k+\ell}$.

Proof. Let

$$
f(u, v)=0
$$

be a real polynomial equation defining $X$, where $u=\left(x_{1}, \ldots, x_{k-1}\right)$ and $v=x_{k}$. Since

$$
X \subset H,
$$

the subset $Y$ of $\mathbb{R}^{k+\ell}$ is defined by the equation

$$
f(u, \rho)=0,
$$

where

$$
\rho=\left(x_{k}^{2}+x_{k+1}^{2}+\cdots+x_{k+\ell}^{2}\right)^{\frac{1}{2}} .
$$

We will now show that (2) can be replaced by a polynomial equation in $x_{1}, \ldots, x_{k-1}$, $x_{k}, \ldots, x_{k+\ell}$. To this end we write

$$
f(u, v)=g\left(u, v^{2}\right)+v h\left(u, v^{2}\right),
$$


where $g$ and $h$ are real polynomials in $(u, v)$. Then (2) is equivalent to

$$
g\left(u, \rho^{2}\right)+\rho h\left(u, \rho^{2}\right)=0,
$$

and in view of (1) also to

$$
\left(g\left(u, \rho^{2}\right)\right)^{2}-\rho^{2}\left(h\left(u, \rho^{2}\right)\right)^{2}=0,
$$

which is a polynomial equation, as required. Consequently, $Y$ is an algebraic subset of $\mathbb{R}^{k+\ell}$.

It is clear that $\psi$ is injective and $\theta: Y \rightarrow X$,

$$
\theta\left(x_{1}, \ldots, x_{k-1}, x_{k}, \ldots, x_{k+\ell}\right)=\left(x_{1}, \ldots, x_{k-1}, \frac{x_{k}}{\rho}, \ldots, \frac{x_{k+\ell}}{\rho}\right),
$$

is the inverse of $\psi: X \rightarrow Y$. By (4),

$$
\rho=-\frac{g\left(x_{1}, \ldots, x_{k-1}, x_{k}^{2}+\cdots+x_{k+\ell}^{2}\right)}{h\left(x_{1}, \ldots, x_{k-1}, x_{k}^{2}+\cdots+x_{k+\ell}^{2}\right)}
$$

for $\left(x_{1}, \ldots, x_{k-1}, x_{k}, \ldots, x_{k+\ell}\right)$ in $Y$, and hence $\theta$ is a regular map. Thus $\psi: X \rightarrow Y$ is a biregular isomorphism.

Assume now that $X$ is projectively closed in $\mathbb{R}^{k}$. We may also assume that the homogeneous form of top degree in $f$, denoted $F$, vanishes only at 0 in $\mathbb{R}^{k}$. Note that $F\left(u, \rho^{2}\right) F\left(u,-\rho^{2}\right)$ is the homogeneous form of top degree in equation (5). This form vanishes only at 0 in $\mathbb{R}^{k+\ell}$, and hence $Y$ is projectively closed in $\mathbb{R}^{k+\ell}$.

Lemma 3.2. The map $g: \mathbb{P}^{2}(\mathbb{C}) \rightarrow \mathbb{P}^{4}(\mathbb{C})$,

$$
g\left(\left(x_{1}: x_{2}: x_{3}\right)\right)=\left(x_{1}^{2}+x_{2}^{2}+x_{3}^{2}: x_{1} x_{2}: x_{1} x_{3}: x_{2} x_{3}: x_{1}^{2}+2 x_{2}^{2}+3 x_{3}^{2}\right),
$$

is an algebraic embedding. In particular, the restriction $f: \mathbb{P}^{2}(\mathbb{R}) \rightarrow \mathbb{P}^{4}(\mathbb{R})$ of $g$ is an algebraic embedding.

Proof. One readily checks that $g$ is injective. Moreover, the (complex) differential of $g$ at each point of $\mathbb{P}^{2}(\mathbb{C})$ is of rank 2. It follows that $g$ is an algebraic embedding, and hence $f$ is an algebraic embedding.

Proof of Theorem 1.1. Let $f: \mathbb{P}^{2}(\mathbb{R}) \rightarrow \mathbb{P}^{4}(\mathbb{R})$ be the algebraic embedding of Lemma 3.2. Note that the image $X=f\left(\mathbb{P}^{2}(\mathbb{R})\right)$ is a projectively closed algebraic subset of $\mathbb{R}^{4} \subset \mathbb{P}^{4}(\mathbb{R})$, contained in the open half-space

$$
\left\{\left(u_{1}, u_{2}, u_{3}, u_{4}\right) \in \mathbb{R}^{4} \mid u_{4}>0\right\} .
$$

Let

$$
\psi: X \times S^{m-2} \longrightarrow \mathbb{R}^{4+(m-2)}=\mathbb{R}^{m+2} \subset \mathbb{P}^{m+2}(\mathbb{R})
$$


be the algebraic embedding of Lemma 3.1 (with $k=4$ and $\ell=m-2$ ). Note that $\psi\left(X \times S^{m-2}\right)$ is projectively closed in $\mathbb{R}^{m+2}$, and hence is an algebraic subset of $\mathbb{P}^{m+2}(\mathbb{R})$.

Clearly, if $i: S^{m-2} \rightarrow S^{m-2}$ is the identity map, then

$$
f \times i: \mathbb{P}^{2}(\mathbb{R}) \times S^{m-2} \longrightarrow X \times S^{m-2}
$$

is a biregular isomorphism. Denoting by $j: \mathbb{P}^{m+2}(\mathbb{R}) \rightarrow \mathbb{P}^{n}(\mathbb{R})$ the standard embedding,

$$
j\left(\left(v_{0}: \ldots: v_{m+2}\right)\right)=\left(v_{0}: \ldots: v_{m+2}: 0: \ldots: 0\right),
$$

we obtain

$$
\varphi=j \circ \psi \circ(f \times i)
$$

which implies that $\varphi$ is an algebraic embedding. In other words, conditions (i) and (ii) are satisfied. Moreover, $M \subset \mathbb{R}^{n} \subseteq \mathbb{P}^{n}(\mathbb{R})$. Since $M$ is nonorientable and $2 m-n \geq 2$, condition (iii) follows from Proposition 2.2.

\section{References}

[1] S. Akbulut and H. King, Transcendental submanifolds of $\mathbb{R}^{n}$. Comment. Math. Helv. 68 (1993), 308-318. Zbl 0806.57017 MR 1214234

[2] S. Akbulut and H. King, Transcendental submanifolds of $\mathbb{R}^{n}$. Comment. Math. Helv. 80 (2005), 427-432. Zbl 1071.57026 MR 2142249

[3] J. Bochnak, M. Buchner and W. Kucharz, Vector bundles over real algebraic varieties. $K$ Theory 3 (1989), 271-298; Erratum, K-Theory 4 (1990), 103. Zbl 0761.14020 MR 1076527

[4] A. Grothendieck, Technique de descente et théorèmes d'existence en géométrie algebrique, I-VI. Seminaire Bourbaki, Exposés 190, 195, 212, 221, 232, 236, 1959-62. Zbl 0234.14007 MR 1603475

[5] J. van Hamel, Algebraic cycles and topology of real algebraic varieties. Dissertation, Vrije Universiteit Amsterdam. CWI Tract. 129, Stichting Mathematisch Centrum, Centrum voor Wiscunde en informatica, Amsterdam 2000. Zbl 0986.14042 MR

[6] R. Hartshorne, Equivalence relations on algebraic cycles and subvarieties of small codimension. Proc. Sympos. Pure Math 29 (1975), 129-164. Zbl 0314.14001 MR 0369359

[7] H. Hironaka, Resolution of singularities of an algebraic variety over a field of characteristic zero. Ann. of Math. 79 (1964), 109-203. Zbl 0122.38603 MR 0199184

[8] H. King, Approximating submanifolds of real projective space by varieties. Topology 15 (1976), 81-85. Zbl 0316.57015 MR 0396572

Received February 28, 2007

Wojciech Kucharz, Department of Mathematics and Statistics, University of New Mexico, Albuquerque, New Mexico 87131-0001, U.S.A.

E-mail: kucharz@math.unm.edu 